\title{
Investigation of wireless optical technology for communication between on-body nodes
}

\author{
L. Chevalier, S. Sahuguede, A. Julien-Vergonjanne \\ XLIM DPT C2S2 UMR CNRS 7252/Labex Sigma-Lim \\ University of Limoges \\ France
}

\author{
P. Combeau, L. Aveneau \\ XLIM DPT SIC UMR CNRS 7252 \\ University of Poitiers \\ France
}

\begin{abstract}
Interferences in Body Area Networks (BANs) are a great challenge in order to ensure a good quality of service in health monitoring systems. BANs are disturbed by several kinds of wireless communications, as mobile cells or Wi-Fi systems. Thus the wireless optical technology could be considered as a solution to reduce the interference amount. The validity of such a solution is examined in terms of transmitted power and data rate. The novelty of this paper is that this is the first time where diffuse optical wireless technology is considered to achieve "on body" communication, using the multiple reflections of optical beams in the patient room.
\end{abstract}

Keywords-Body Area Network; wireless optical communication; outage probability;

\section{INTRODUCTION}

Body Area network (BAN) is a recent and promising technology particularly investigated in the field of health monitoring applications. A BAN standard is discussed by the IEEE 802.15.6 Task group to define wireless communications on, in or around the human body [1]. BANs differ from the classical wireless networks in terms of size and power consumption in order to be wearable and to ensure a satisfying lifetime. Moreover, they have to coexist with several wireless networks while maintaining the high quality of service needed by the transmission of health information [2]. Among wireless technologies, UWB (Ultra Wide Band) is a good candidate thanks to the low power spectrum density and high capacity of transmission it offers [1-3]. However, this technology still suffers from interferences due to the other BANs and various wireless networks existing in the patient's environment. On top of that, the propagation of the EM (electromagnetic) waves on the human body is very complex, difficult to model and leads to a waste of performances of the UWB systems [4] for "on-body" communications because of the limited power available defined by the legislation [5].

In this paper, an alternative solution for BANs is proposed, using optical wireless technology to realize an "on-body to onbody" communication. The optical wireless communications have been intensively studied for both indoor and outdoor applications in recent years, because this technology offers several advantages such as high bandwidth, low complexity, and high security [6-12]. Even if it has been investigated in the field of sensor networks [9-10], as far as known, it is still not the case concerning the "on-body" networks. Our contribution is to determine the wireless optical technology performances for BANs compared to RF UWB ones. For this purpose, the same room setting and human type as those used to measure and model the standard RF channel for "on-body to on-body" communication, known as CM3 [13], will be taken into account. Then, the gain distribution on the human body of the $\mathrm{RF}$ and optical channels will be determined by simulation. Thus, it will lead to obtain the outage probability of each channel, in the aim to compare their performances.

The paper is organized as follows. Section 2 describes the $\mathrm{RF}$ and the optical channels, and highlights their differences. Their respective performances, presented here in terms of outage probability are presented in section 3 .

\section{DESCRIPTION OF THE STUDIED SYSTEM}

\section{A. Context}

In this part, the channel models for UWB and optical wireless techniques are defined and compared. For this aim, the same indoor environment as the one described in the IEEE 802.15.6 for the on body communication (CM3) is considered [13]. The CM3 channel was determined from measurements made in a hospital room as described in Figure 1.
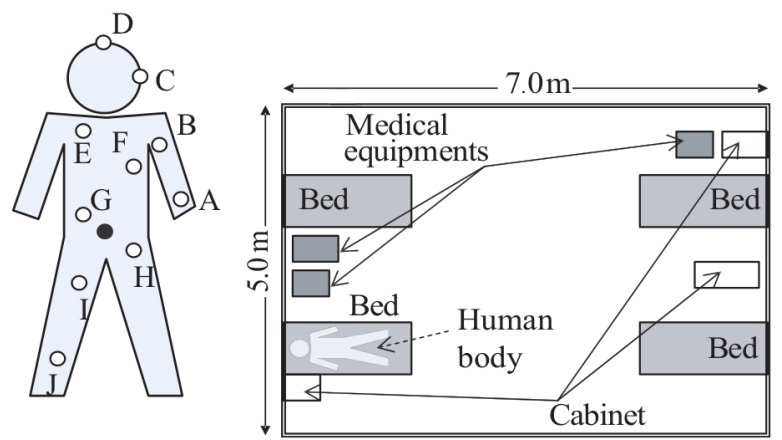

Fig 1. Patient and hospital room 
The human body considered is a male, with a height of $171 \mathrm{~cm}$ and lying on his bed, and the measurements have been carried out on the frequency band [3.1-10.6] $\mathrm{GHz}$. The point $\mathrm{G}$, represented in figure 1 , corresponds to the master node of the BAN, and the others are the different positions of transmitters.

\section{B. CM3 channel description.}

The CM3 channel is obtained from measurements between 2 nodes: the first one is placed at the point $\mathrm{G}$, and the second one is taken at different positions (A to $\mathrm{F}$ and $\mathrm{H}$ to $\mathrm{J}$ ) seen in figure 1. They provide the Power Delay Profile (PDP) model, described in table 1, and the pathloss one (1),

$$
\mathrm{PL}(\mathrm{d})=\mathrm{a} \cdot \log _{10}(\mathrm{~d})+\mathrm{b}+\mathrm{N}
$$

with $\mathrm{d}$ the distance between two nodes in $\mathrm{mm}$, $\mathrm{a}$ and $\mathrm{b}$ respectively equal to 19.2 and 3.38 , and $\mathrm{N}$ a normally distributed variable with zero mean and standard deviation $\sigma_{\mathrm{N}}$ of $4.40 \mathrm{~dB}$ [13].

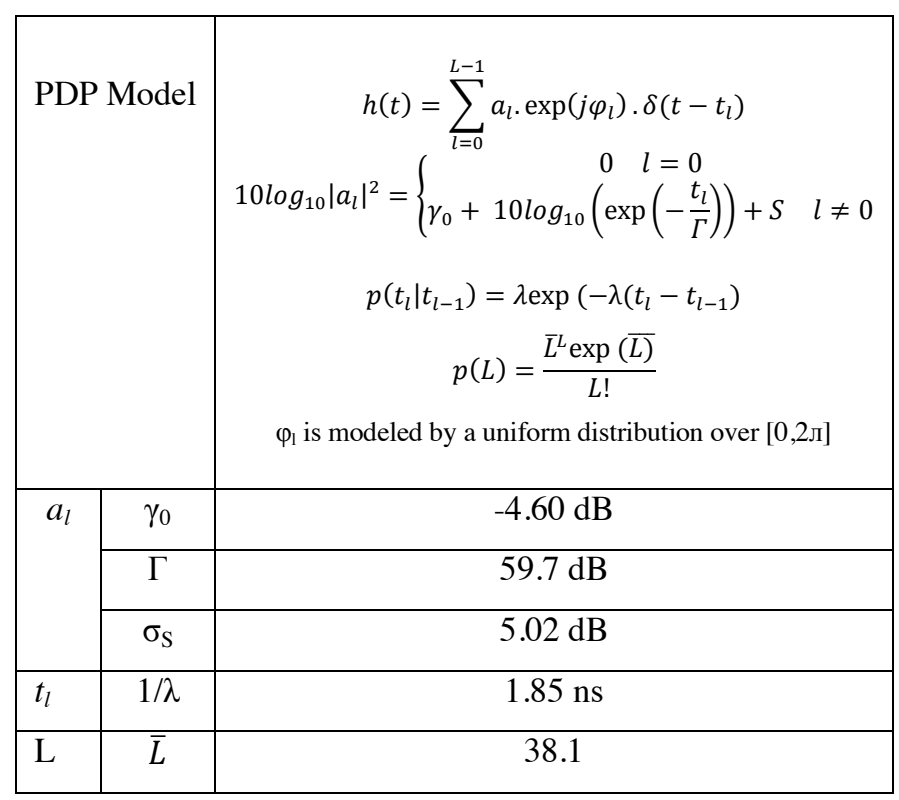

table 1. PDP model

- $\quad a_{l}$ : path amplitude for the $l$-th path

- $\mathrm{t}_{l}$ : path arrival time for the $l$-th path

- $\Phi_{1}$ : phase for the $l$-th path

- $\mathrm{L}$ : the number of the arrival paths

- $\quad \delta(\mathrm{t})$ : the Dirac function

- $\quad \Gamma$ : an exponential decay with a Rician factor $\gamma_{0}$

- S: a normal distribution with zero-mean and standard deviation of $\sigma_{\mathrm{S}}$

- $\lambda$ : path arrival rate

- $\bar{L}$ : the average $\mathrm{L}$ number

- $\mathrm{h}(\mathrm{t})$ : the general formulation of the PDP
The simulation of the CM3 channel model has been reported in figure 2 , in terms of PDP and pathloss. These two results represent only one realization of the model. In order to get average results, this has to be done several hundred times, in order to take into account all the possibilities.

Practice has shown that a hundred times was the minimum to obtain similar results between several simulations. In this paper, a number of five hundred realizations of the CM3 channel by RF simulation is assumed. Thus, it is possible to evaluate the average depth of the channel (64 ns), and to verify that the average number of paths is effectively around 38 , as presented in figure 3 .
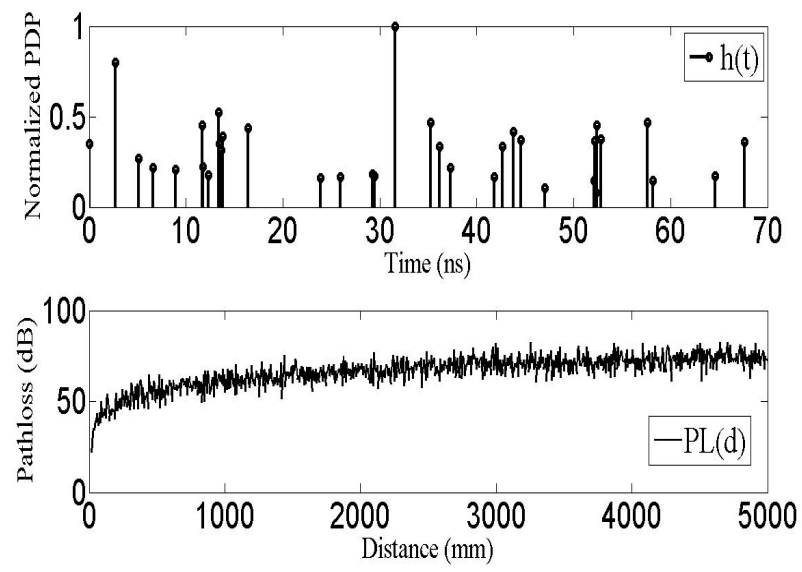

Fig 2. channel simulation
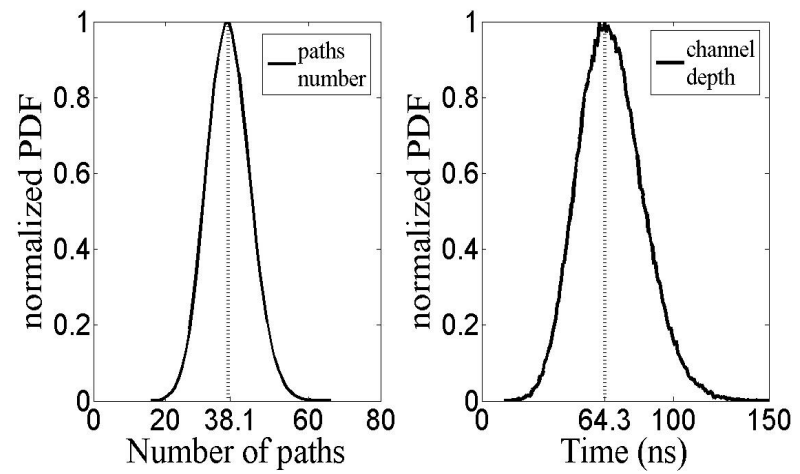

Fig 3. PDF of the paths number and the channel depth of CM3

As said previously, the aim here is to get the gain distribution of the CM3 channel on the human body. Thus, instead of the 10 sensor positions shown in figure 1, a uniform distribution of two hundred thousand positions on the human body is used.

On top of that, it is necessary to take into account the criteria of low power consumption and low complexity of the wearable devices. Thus, instead of a Rake receiver which could use multiple paths, a simpler reception system, considering only the dominant path of the channel, is used. It leads to the gain distribution $\mathrm{G}_{0 \mathrm{CM} 3}$ provided by the dominant path, represented in figure 4 . 


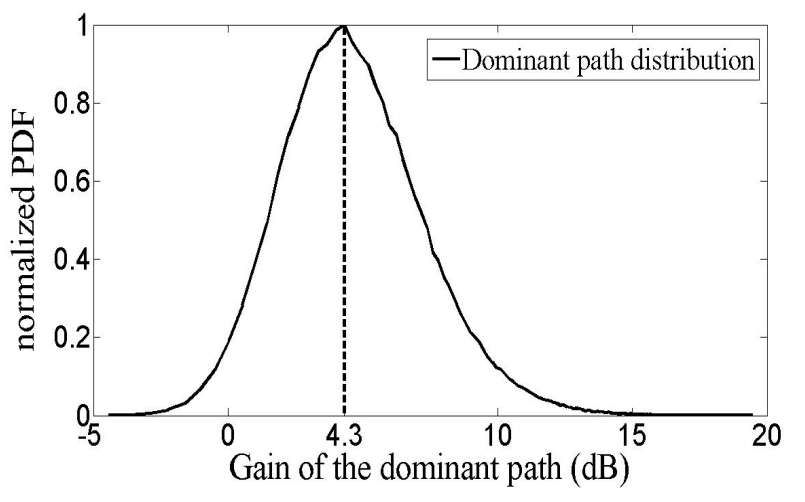

Fig 4. Distribution of $\mathrm{G}_{0 \mathrm{CM} 3}$

Then, in order to get the distribution of $\mathrm{H}_{0 \mathrm{CM} 3}$ (the $\mathrm{RF}$ channel gain), the following formula is used at each position of the transmitter on the human body:

$$
H_{0 C M 3}=G_{0 C M 3}+P L(d)
$$

The results reported on figure 6 show a wide distribution because of the large variation of the pathloss from 40 to $70 \mathrm{~dB}$ on the human body, compared to $\mathrm{G}_{0 \mathrm{CM} 3}$, varying from -3 to 15 $\mathrm{dB}$. In this case, the distance between the transmitters and the master node predominates because the strongest link is the direct one.

\section{Optical channel description}

To perform on body sensor communications, a diffuse optical communication [11,12] has been chosen for the following reasons:

- A direct link between two nodes is difficult to envisage, because of the variety of the human body morphologies. A typical example is the difference between men and women for the $[\mathrm{E}, \mathrm{G}]$ link shown in figure 1, where the chest could be an obstacle.

- The complexity required in order to achieve a wearable system using a LOS (Line Of Sight) solution requiring a perfect alignment, and adapted to the small movements of the body on the bed, or to a moving body in the room, is not adapted to the simplicity criterion of BANs.

Thus, a transmission node projecting an optical beam on the environment surfaces is considered. Then, the reflected signals are collected by the optical receiver at the master node $\mathrm{G}$, as represented in figure 5. The optical receiver is supposed to have a physical surface of $1 \mathrm{~cm}^{2}$, a responsivity $\mathrm{S}=0.55 \mathrm{~A} / \mathrm{W}$, and a diffuse Field of View (FOV) of 70 degrees [12]. The transmission node is considered as a Lambertian transmitter, with a wavelength of $850 \mathrm{~nm}$, and a half-power angle of 60 degrees, using an On-Off Keying (OOK) modulation scheme [12].

In order to obtain results that can be compared to the CM3 channel ones, the same position distribution of the transmitters cited before is used. On top of that, the same room as the one presented in figure 1 is modeled in order to provide a channel in the same conditions, as presented in figure 5 .

To obtain the optical gain $\mathrm{H}_{\text {0optic }}$ distribution, the software RaPSor [14] is used. It is a ray-based simulator developed at the Xlim Laboratory of the Poitiers University. It offers the possibility to simulate the propagation of a wave according to several physical configurations from the hypothesis of high frequency approximation. It is based on techniques such as a classical ray launching associated to Monte Carlo algorithm.

The simulation results are provided in figure 6. All the reflective surfaces of the room have been considered, in a first approach, with the same reflection coefficient $\rho=0.8$ [15]. It can be noted that the optical gain $\mathrm{H}_{0 \text { optic }}$ distribution spreading is very short compared to the RF one, because of the reflections linked to the diffuse propagation used. Actually, the communication between the two nodes is done through the multiple reflections, and the distance traveled by the optical beams is similar for each of them, leading to small variations of the pathloss around the different transmitters positions.

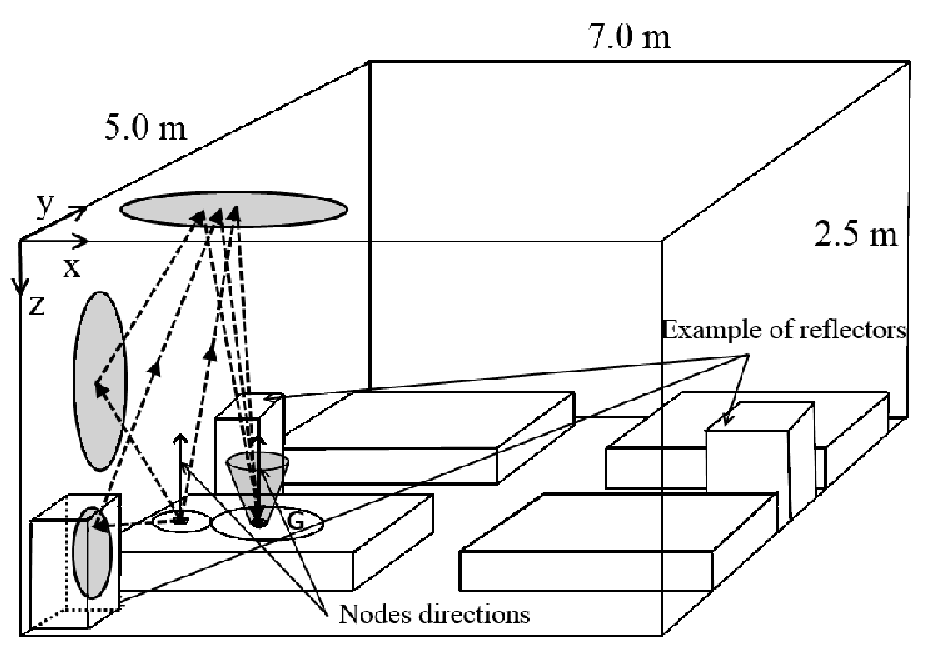

Fig 5. Patient room modeling for optical channel simulation

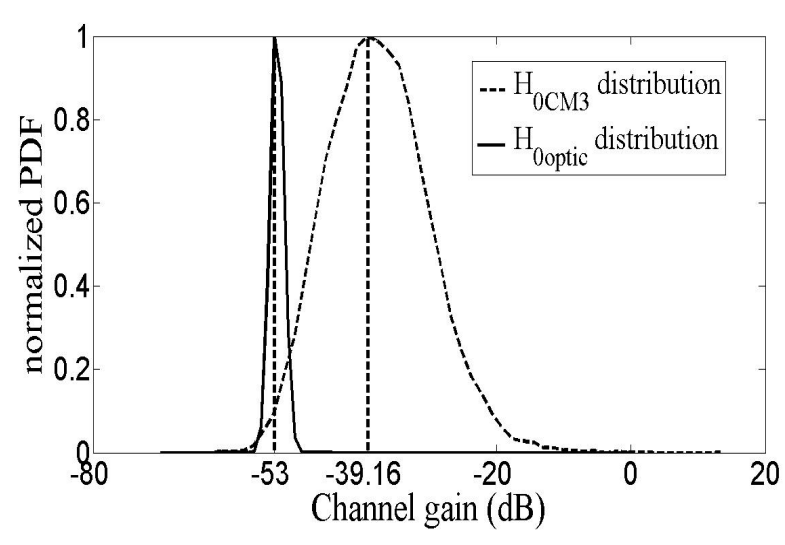

Fig 6. Optical and RF gains distributions 
Moreover, $\mathrm{H}_{0 \mathrm{CM} 3}$ presents a higher mean value than $\mathrm{H}_{0 \text { optic }}$ (a difference of $13.8 \mathrm{~dB}$ ). Thus, this means that in order to ensure the QoS needed in the health monitoring application, the optical solution need to transmit more power than the RF one, as detailed in the following section.

\section{OUTAGE PROBABILITY}

\section{A. Definition}

Because of the wide distribution of the RF channel gain, shown in figure 6 , using an average bit error rate (BER) as performance criterion wouldn't be enough. Thus, the outage probability is used in this paper. We consider an outage if the SNR (signal noise ratio) is lower than a $\mathrm{SNR}_{0}$ which corresponds to the desired quality of service. The outage probability can thus be written as [12]:

$$
\operatorname{Pout}\left(S N R_{0}\right)=\operatorname{Pr}\left(S N R<S N R_{0}\right)
$$

The SNRs for optical and RF cases are calculated as follow:

$$
\begin{gathered}
S N R_{R F, d B}=P_{t R F}+G_{0 C M 3}-P L(d)-N_{R F} \\
S N R_{O p t, d B}=10 \log _{10}\left(\frac{2 S^{2} P_{t o p t i c}{ }^{2} H_{0 o p t i c}{ }^{2}}{N_{0} D_{b}}\right)
\end{gathered}
$$

With $\mathrm{N}_{0}$ the classical ambient light noise $\left(6.4 .10^{-23} \mathrm{~W} / \mathrm{Hz}\right), \mathrm{D}_{\mathrm{b}}$ the data rate in bits/s, $\mathrm{N}_{\mathrm{RF}}$ a typical RF noise power $(-84 \mathrm{dBm})$, $\mathrm{P}_{\mathrm{tRF}}$ the $\mathrm{RF}$ transmission power, and $\mathrm{P}_{\text {toptic }}$ the optical transmission power.

Then, we can define the outage probability as the cumulative density function (CDF) of the SNR, evaluated for $\mathrm{SNR}_{0}$.

In order to get the distribution of the SNR for both cases, we must define the maximum transmission powers available $\mathrm{P}_{\mathrm{tRF}}$ and $\mathrm{P}_{\text {toptic. }}$

Concerning the RF field, the power is limited [5] by the federal radio commission $(\mathrm{FCC}$ ) at $-41.3 \mathrm{dBm} / \mathrm{MHz}$. It leads to a maximum transmission power of $-2.5 \mathrm{dBm}$ in the UWB band. Considering a typical pulse, such as a Gaussian wavelet with a width of $2 \mathrm{~ns}$, a $-10 \mathrm{~dB}$ bandwidth [3.1-4.5] GHz and centered on $3.8 \mathrm{GHz}$, the available frequency band won't be totally used, and then we can assume a maximum transmission power of $\mathrm{P}_{\mathrm{tRF}} \#-9.5 \mathrm{dBm}(0.11 \mathrm{~mW})$ for more realistic simulations.

Regarding the optical case, the diffuse optical propagation is used, for a wavelength of $875 \mathrm{~nm}$. Thus, standards define a maximum radiated intensity of $95.5 \mathrm{~mW} / \mathrm{sr}$ [16]. For a halfpower angle of 60 degrees, the maximum transmission power is $\mathrm{P}_{\text {toptic }} \# 300 \mathrm{~mW}$ [14].

\section{B. Results}

Firstly, it is valuable to determine the outage probability for each case, at the maximum transmission power available. The simulation results are shown in figure 7 , for an example data rate of $4.2 \mathrm{Mb} / \mathrm{s}$.

We can remark that wireless optical technology performances are better than RF ones in terms of outage probability for a given $\mathrm{SNR}_{0}$
These results show that, despite the advantage presented in terms of gain by the RF channel, the optical technology can overcome this issue thanks to the higher transmission power available.

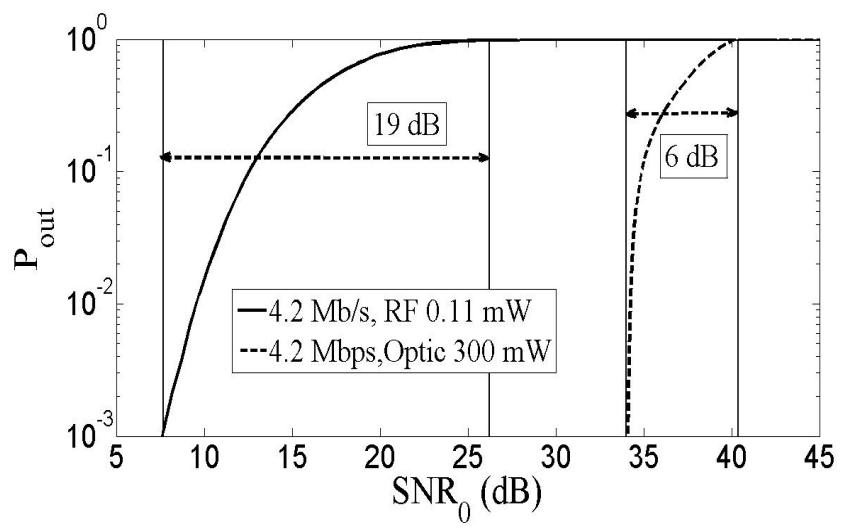

Fig 7. Optical and RF outage probabilities at maximum transmission power

Besides, it can be seen that RF curve has a variation range of $19 \mathrm{~dB}$ from an outage probability of $10^{-3}$ to a value of 1 , whereas it is only $6 \mathrm{~dB}$ for the optical link. This means that optical wireless link performance can be degraded more rapidly than the $\mathrm{RF}$ one.

Thus, the point is now to find the lowest optical power needed to reach the same performances with optical technology as with the RF one. The simulation results are shown figure 8 , for a targeted $\mathrm{P}_{\text {out }}$ of $10^{-3}$.

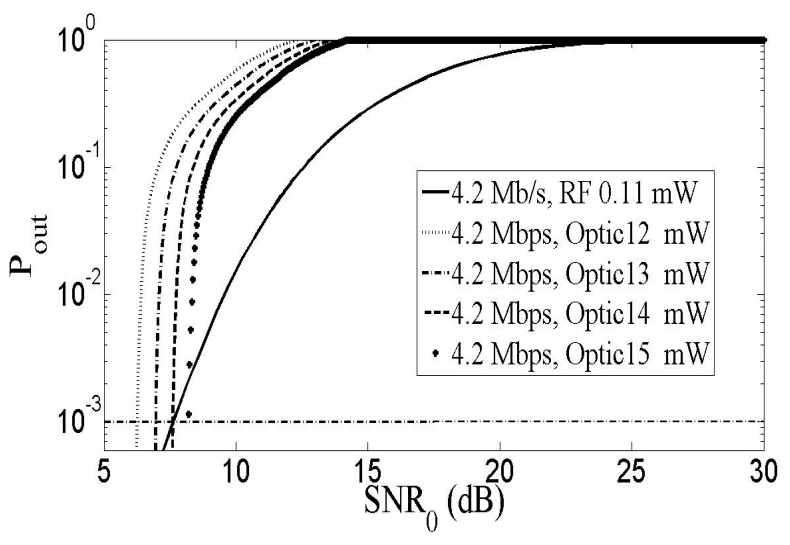

Fig 8. Outage probability for various optical transmission powers

We can deduce from these results that an optical transmission power around $14 \mathrm{~mW}$ is sufficient to obtain the same QoS as the RF solution with $\mathrm{P}_{\mathrm{tRF}} \# 0.11 \mathrm{~mW}$. Compared to the maximum available power of $300 \mathrm{~mW}$, this value of $14 \mathrm{~mW}$ shows that the optical wireless technology has a lot more flexibility in terms of power and therefore performance, than RF technology. 
In addition, to respect the QoS needed by health monitoring applications corresponding to a BER of $10^{-9}$, we need to aim a $\mathrm{SNR}_{0}$ of $15.6 \mathrm{~dB}$ with a Pout of $10^{-3}$, and a data rate of $1 \mathrm{Mb} / \mathrm{s}$ [2]. Thus, the minimum optical transmission power has to be determined. The simulation results are provided in figure 9.

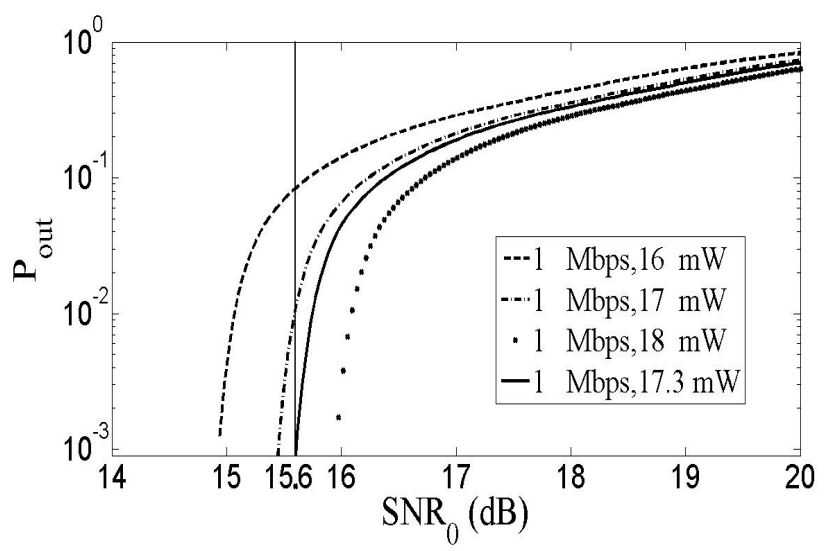

Fig 9. Optical outage probability for various data rates

These results show that a minimum optical transmission power of $17.3 \mathrm{~mW}$ is required, which is as previously significantly lower than the maximum power available for wireless optical communications.

\section{CONCLUSION}

In this paper, the relevancy of studying the optical wireless technology for body area networks has been shown. However, several points remain to be studied.

The system presented in this paper only concern the performances of one link between two nodes, without taking into account the impact of the presence of other transmitters. Therefore, the comparison between optical and RF performances is not complete.

To continue, the simulation can be improved, by considering more realistic reflection coefficients for the different surfaces used to model the room.

To finish, the transmission power needed to ensure the same performances in optical communications as RF ones is still higher than the typical value used in RF. This can have a significant impact on the lifetime of the system. However, the potential performance of wireless optical technology applied to BANs deserves further investigations.

\section{REFERENCES}

[1] IEEE 802.15.6 standard for Local and metropolitan area networks - Part 15.6: Wireless Body Area Networks, 2012.

[2] S. Ullah, P. Khan, N. Ullah, S. Saleem, H. Higgins, and K.S. Kwak, "A review of wireless body area networks for medical applications", Int'1 J. Communications Network and System Sciences, 2010.

[3] C. Keong Ho, T.S.P. See, M.R. Yuce, "An ultra-wideband wireless body area network: Evaluation in static and dynamic channel conditions", Sensors and Actuators A 180,pp137-147, april 2012.

[4] A. Lea, P. Hui, J. Ollikainen, R. Vaughan, "Propagation between on body antennas", IEEE trans. In antennas and propagation, Vol. 57, No.11, pp. 3619-3627, November 2009.

[5] FCC. First report and order regarding UWB transmission. Technical report ET docket 98-153, Federal Communication Commission, Washington, D.C. 20554, 14 February 2002.

[6] J.M. Kahn, and J.R. Barry, "Wireless infrared communications", Proceedings of the IEEE, Vol. 25, PP. 1702-1710, 2007.

[7] J.B. Carruthers, "Wireless infrared communications. Wiley Encyclopedia of telecommunications", Wiley, New York, 2003.

[8] K. Borah, A.C. Boucvalas, C.C Davis, S. Hranilovic, K. Yiannopoulos, "A review of communication-oriented optical wireless systems", EURASIP J. Wirel. Commun. Netw. 91, doi:10.1186/1687-1499-201291.

[9] J. Fadlullah, and M. Kavehrad, "Indoor high-bandwidth optical wireless links for sensor networks", Journal of lightwave technology, Vol. 28, N0. 21, November 1, 2010.

[10] S. Deng, J. Liao, Z.R. Huang, M. Hella, and K. Connor, "Wireless connections of sensor network using RF and free space optical links", Proceedings of SPIE, Vol. 6773, pp. 677307-677307-11, 2007.

[11] H. Elgala, R. Mesleh, and H. Haas, "Indoor optical wireless communication: potential and state-of-the-art", Communications Magazine, IEEE DOI - 10.1109/MCOM.2011.6011734, vol. 49, no. 9, pp. 56-62, 2011.

[12] S.S. Torkestani, N. Barbot, S. Sahugude, A. Julien-Vergonjanne, J.P. Cances, "Performance and transmission power bound analysis for optical wireless based mobile healthcare applications", Personal Indoor and Mobile Radio Communications (PIMRC), 2011 IEEE $22^{\text {nd }}$ International Symposium on, 2011, pp. 2198-2202.

[13] K. Takizawa, T. Aoyagi, and R. Kohno, "Channel modeling and performance evaluation of UWB-based wireless body area networks", Proc. IEEE ICC, 1-5, 2009.

[14] RaPSoR, Ray Propagation SimulatoR, http://rapsor.sourceforge.net/index.

[15] J.R. Barry, J.M. Khan, W.J. Krause, E.A. Lee, and D.G. Messerschmitt, "Simulation of multipath impulse response for indoor wireless optical channels", IEEE journal on selected areas in communications, Vol. 11, No. 3, 1993.

[16] "IEC 60825-1, safety of laser products-part 1: equipment classification, requirements, and user's guide",2001, 1.2 Edition. 